\title{
Diagnóstico in vitro y en campo de resistencia antihelmíntica en nematodos gastrointestinales de pequeños rumiantes
}

\author{
In vitro and field diagnosis of anthelmintic resistance in gastrointestinal nematodes \\ of small ruminants \\ R González-Garduño ${ }^{\mathrm{a}^{*}}$, ME López-Arellano ${ }^{\mathrm{b}}$, N Ojeda-Robertos ${ }^{\mathrm{c}}$, \\ E Liébano-Hernández ${ }^{b}$, P Mendoza-de Gives ${ }^{b}$ \\ ${ }^{a}$ Unidad Regional Universitaria Sursureste, Universidad Autónoma de Chapingo, Teapa, Tabasco, México. \\ bInstituto Nacional de Investigaciones Forestales, Agrícolas y Pecuarias, Centro Nacional de Investigación Disciplinaria \\ en Parasitología Veterinaria, Jiutepec, Morelos, México. \\ cDivisión Académica de Ciencias Agropecuarias, Universidad Juárez Autónoma de Tabasco, Tabasco, México.
}

\begin{abstract}
SUMMARY
The problem of anthelmintic resistance in ovine trichostrongyles is growing in tropical areas such as Chiapas, Mexico. Hair-sheep breeds are frequently used by farmers because of their climate adaptation, however, anthelmintic resistance incidence requires more sensitive methods of diagnosis in this region in order to apply selective treatments. The aim of this study was to evaluate in vitro and in vivo the relative efficacy of the three main anthelmintics used to control gastrointestinal nematodes (GIN) in hair sheep. Naturally infected ewes and lambs experimentally infected with 100 larvae $\left(\mathrm{L}_{3}\right)$ of Haemonchus contortus and Cooperia curticei per kg body weight were used. Sheep were treated with $10 \mathrm{mg} \mathrm{kg}^{-1} \mathrm{albendazole,} 7,5 \mathrm{mg} \mathrm{kg}^{-1}$ levamisole or $0,2 \mathrm{mg} \mathrm{kg}^{-1}$ ivermectin. Positive faecal samples were cultured to obtain infective larvae $\left(\mathrm{L}_{3}\right)$. Larvae of $C$. curticei and $H$. contortus were tested in vitro and data were analyzed using the PROBIT procedure. Also, the relative anthelmintic efficacy was evaluated by faecal egg reduction test (FECRT). The results of the FECRT showed $30 \%$ of levamisole efficacy while efficacies of and $87 \%, 64 \%$ and $65 \%$ were observed for ivermectin, albendazole and commercial combination of ivermectin + levamisole $(\mathrm{Iv}+\mathrm{L})$, respectively. Problems of anthelmintic resistance were manly showed with the mix $\mathrm{Iv}+\mathrm{L}$. The lethal dose $\left(\mathrm{LD}_{50}\right)$ to albendazole, levamisole and ivermectin estimated after $12 \mathrm{~h}$ were $38,8,9,5 \mathrm{and} 0,41 \mathrm{mg}$. These result show anthelmintic resistance problems in hair sheep using the three main commercial drugs and for the combination of Iv+L in the tropical region of Chiapas, Mexico.
\end{abstract}

Key words: hair sheep, gastrointestinal nematodes, anthelmintic resistance, Probit test.

\section{RESUMEN}

El problema de la resistencia antihelmíntica en trichostrongílidos se incrementa en la región tropical, tal es el caso de Chiapas, México. Las razas de ovinos de pelo son utilizadas frecuentemente por lo productores debido a su adaptación climática. Sin embargo, la incidencia de resistencia antihelmíntica requiere de métodos más sensibles para el diagnóstico y así aplicar los tratamientos adecuados. El objetivo del presente estudio fue evaluar la eficacia in vitro e in vivo de los tres principales antihelmínticos en el control de nematodos gastrointestinales (ngi) en ovinos de pelo. Se utilizaron ovejas con infección natural y corderos infectados experimentalmente con 100 larvas $\left(\mathrm{L}_{3}\right)$ de Haemonchus contortus y Cooperia curticei por $\mathrm{kg}$ de peso. Los animales fueron tratados con albendazol a $10 \mathrm{mg} / \mathrm{kg}^{-1}$, levamisol a 7,5 mg/ $\mathrm{kg}^{-1} \mathrm{o}$ ivermectina a $0,2 \mathrm{mg} / \mathrm{kg}^{-1}$. Las muestras fecales se cultivaron para obtener larvas infectantes $\left(\mathrm{L}_{3}\right)$, estas se sometieron a la prueba in vitro y los datos se analizaron con el procedimiento Probit. También se evaluó la eficiencia de los antihelmínticos con la prueba de reducción del número de huevos de ngi (PRCFH). Los resultados de PRCFH mostraron una eficacia de $30 \%$ para levamisol, mientras que con ivermectina, albendazol y la aplicación de ivermectina + levamisol (Iv+L) hubo una eficacia de $87 \%$, $64 \%$ y $65 \%$, respectivamente. Se observaron problemas de resistencia antihelmíntica en la aplicación de Iv+L. La estimación de la dosis letal $\left(\mathrm{DL}_{50}\right)$ para albendazol, levamisol e ivermectina a las 12 horas fue de 38,8, 9,5 y 0,41 mg, respectivamente. Los resultados muestran que hubo problemas de resistencia antihelmíntica en los ovinos de pelo usando los tres principales desparasitantes comerciales y para la combinación de Iv+L en la región tropical de Chiapas, México.

Palabras clave: ovinos de pelo, nematodos, resistencia antihelmíntica, prueba Probit.

\section{INTRODUCCIÓN}

La resistencia antihelmíntica se define como la disminución en la efectividad de un desparasitante contra una población de parásitos susceptibles utilizando la dosis

Aceptado: 21.11.2013.

* Apartado Postal No. 29, Teapa, Tabasco, CP. 86800, México; robgardu@hotmail.com terapéutica (Jabbar y col 2006). Este problema es ampliamente conocido en nematodos gastrointestinales (ngi) que afectan a una gran variedad de animales domésticos (cerdos, equinos y rumiantes), lo que se atribuye al uso continuo de los desparasitantes (Taylor y col 2002). Como resultado de la resistencia antihelmíntica se seleccionan grupos de nematodos con alelos resistentes, y los sobrevivientes se recombinan formando poblaciones que muestran fenotipo de tolerancia antihelmíntica (Sangster 2001). Esta situación 
se ha convertido en un problema mundial, al presentarse tanto en países con clima templado (Chartier y col 1998) y en aquellos con condiciones climatológicas cálidas en los trópicos (Torres-Acosta y col 2003, Encalada-Mena y col 2008).

Los nematodos gastrointestinales han tenido una rápida evolución y desarrollo de resistencia contra los principales productos químicos utilizados en su control (Taylor y col 2002), como son: bencimidazoles, imidazotiazoles y lactonas macrocíclicas (Arece y col 2004, Papadopoulos 2008) e incluso a las combinaciones de antihelmínticos contra cepas resistentes de Haemonchus contortus. El uso de varios fármacos a la vez puede ser una solución temporal, sin embargo, el problema de resistencia se genera rápidamente, y una vez que se establece para ambos productos causa resistencia múltiple (Von Samson-Himmelstjerna 2005, Coles y col 2006).

De los métodos para detectar resistencia antihelmíntica, los más utilizados han sido las pruebas in vitro y la prueba de reducción del conteo fecal de huevos de nematodos (PRCFH). Esta última es sencilla, se puede aplicar a cualquier tipo de fármaco y mide la efectividad in vivo de los antihelmínticos (Coles y col 1992, Coles y col 2006). Es capaz de detectar resistencia cuando la frecuencia de los alelos mutantes con genotipo de resistencia supera el $25 \%$ (Sangster 2001). En contraste, las pruebas in vitro son más sensibles, pero se requiere de personal con experiencia y material biológico. A pesar de las desventajas, las pruebas in vitro aportan información relevante para discriminar aislamientos de ngi resistentes y susceptibles (Coles y col 2006).

La rápida dispersión de ngi resistentes a nivel mundial ha puesto en riesgo la sustentabilidad de las estrategias de control, debido al retardo de la conversión de poblaciones resistentes a susceptibles mediante el manejo (Sangster 2001). Ante la dificultad de no contar con nuevas familias de antihelmínticos es necesario establecer otras formas de diagnóstico y control de los ngi (Molento 2009).

La amplia difusión de cruzamientos con razas especializadas en producción de carne como la Katahdin y Dorper, distribuidas en los estados de Veracruz, Tabasco y Chiapas debido a su adaptabilidad a climas cálidos (Vilaboa-Arroniz y col 2010), pero con mayor susceptibilidad a nematodos gastrointestinales (ngi) es que la raza Pelibuey ha originado un alto uso de antihelmínticos. Por tal situación el objetivo del presente estudio fue evaluar la eficacia in vitro e in vivo de los tres principales antihelmínticos en el control de ngi en ovinos de pelo.

\section{MATERIAL Y MÉTODOS}

\section{LOCALIZACIÓN}

El estudio de eficacia y resistencia antihelmíntica se desarrolló en una unidad de producción de ovinos en Pueblo Nuevo, Municipio de Salto de Agua, Chiapas, México. La unidad se localiza a $85 \mathrm{msnm}$ con coordenadas $17^{\circ} 34^{\prime}$ de latitud norte y $92^{\circ} 29^{\prime}$ de longitud oeste. El clima de la región está clasificado como Af (m) w”(i’)g, cálido húmedo con lluvias todo el año, temperatura promedio anual de $26,6^{\circ} \mathrm{C}$ y precipitación de 3.289,1 mm (García 1988). El proceso y análisis de las muestras biológicas se realizó en la Unidad Regional Universitaria Sursureste de la Universidad Autónoma Chapingo (URUSSE-UACh), ubicada en Teapa, Tabasco.

\section{MANEJO DE LOS ANIMALES}

El rebaño utilizado estaba formado por 41ovejas de pelo, cruza Katahdin x Pelibuey que se encontraban en pastoreo rotacional en potreros de Estrella de África (Cynodon plectostachyus) y pasto Humidícola (Brachiaria humidicola). Los potreros estaban contaminados con larvas de $H$. contortus y $C$. curticei por el pastoreo de ovinos. De forma adicional se utilizaron 24 corderos Pelibuey que se adquirieron en el Centro de Capacitación y Reproducción de Especies Menores (CECAREM) y se criaron en estabulación, libres de ngi. Los corderos fueron infectados experimentalmente con $100 \mathrm{~L}_{3}$ por $\mathrm{kg}$ de peso vivo (PV) de la mezcla de $H$. contortus y $C$. curticei en proporción de 60 y $40 \%$, respectivamente, obtenidos de coprocultivos de ovejas de la misma unidad de producción.

\section{EFICACIA Y RESISTENCIA ANTIHELMÍNTICA}

El rebaño estuvo sometido a un programa de desparasitación selectiva para el control de ngi. Este consistió en desparasitar a las ovejas con conteos fecales mayores de 1.000 hpg y en estos animales se determinó la eficacia de los tres principales antihelmínticos comerciales, los que fueron aplicados a las dosis recomendadas por el fabricante (ivermectina $0,2 \mathrm{mg} / \mathrm{kg}^{-1} \mathrm{PV}$, albendazol $10 \mathrm{mg} /$ $\mathrm{kg}^{-1} \mathrm{PV}$ y levamisol 7,5 mg/ $\mathrm{kg}^{-1} \mathrm{PV}$ ). También se utilizó la combinación de ivermectina + levamisol (Iv+Lev). La terapia antihelmíntica se administró durante la época de lluvias y vientos cuando ocurrió la mayor prevalencia de ngi (junio-diciembre de 2011). Los productos se aplicaron de manera secuencial en diferente fecha a grupos de 7 a 14 animales. Por otra parte, también se confirmó la carga parasitaria en los corderos del Cecarem infectados experimentalmente, y se aplicó albendazol a razón de 10 $\mathrm{mg} / \mathrm{kg}^{-1} \mathrm{PV}$ a aquellos animales con más de 300 hpg, por lo que el número de ovejas y corderos tratados con albendazol fue de 26 animales.

Tanto en las ovejas como en los corderos se realizó el monitoreo de la carga parasitaria entre los 10 y 14 días postratamiento, tomando muestras de heces directamente del recto de cada animal para determinar el número de huevos de ngi mediante la técnica de McMaster (Thienpont y col 1986).

Se calculó el porcentaje de eficacia como la diferencia en el porcentaje entre los conteos después (1) y antes (0) del 
tratamiento (100 - (hpg $1 /$ hpg 0)*100) por animal como repetición (Kochapakdee y col 1995). La comparación entre los tratamientos se realizó transformando a raíz cuadrada la variable (hpg 1/hpg 0) y se comprobó la normalidad de la variable con la prueba de Kolmogorov-Smirnov y Cramer-Von Mises del procedimiento univariado del SAS (SAS 1999). El análisis estadístico se realizó con el procedimiento GLM del SAS (SAS 1999) y la prueba de separación de medias con el test de Tukey.

En 16 corderos con conteos superiores a los $300 \mathrm{hpg}$ y con promedio general de $1.446 \pm 963 \mathrm{hpg}$, infectados de manera experimental con $100 \mathrm{~L}_{3}$ por $\mathrm{kg}$ de peso vivo de un cultivo mixto de $H$. contortus y $C$. curticei, se realizó la prueba de reducción del conteo fecal de huevos de ngi en heces (PRCFH) para el diagnóstico de resistencia antihelmíntica, siguiendo las recomendaciones de la Asociación Mundial para el Avance de Parasitología Veterinaria (WAAVP, Coles y col 2006). Se formaron dos grupos de ocho corderos, el control sin tratamiento antihelmíntico y el tratado con $0,2 \mathrm{mg} / \mathrm{kg}^{-1}$ de ivermectina y $10 \mathrm{mg} / \mathrm{kg}^{-1}$ de levamisol de manera simultánea $(\mathrm{Iv}+\mathrm{L})$.

Con los datos obtenidos de hpg se calculó la media aritmética, intervalo de confianza al $95 \%$ y porcentaje de reducción (Coles y col 1992) de acuerdo con la siguiente ecuación:

$$
\operatorname{PHR}=100\left(1-\frac{\overline{X t}}{\overline{X c}}\right)
$$

Donde: $\mathrm{PRH}=$ Porcentaje de reducción del número de huevos de nematodos, $\overline{X t}=$ Promedio de hpg del grupo tratado, $\overline{X c}=$ Promedio de hpg del grupo control.

\section{PRUEBA IN VITRO}

La prueba in vitro se realizó mediante la técnica de migración larval (Wagland y col 1992) con observación directa de la motilidad. El efecto de los antihelmínticos se evaluó basado en el siguiente criterio: larvas muertas, aquellas que se observaron rectas y sin movimiento, mientras que las larvas vivas se clasificaron en dos categorías: enrolladas y en movimiento. Se utilizó un cultivo mixto de $\mathrm{L}_{3}$ de $H$. contortus y C. curticei (60:40) provenientes de los ovinos infectados en pastoreo de la unidad de producción de Chiapas (Aislamiento Ch). Asimismo, se utilizó un aislamiento de $H$. contortus resistente a bencimidazoles como material de referencia (CENID-PAVET, INIFAP). Después de siete días de cultivo se recuperaron las larvas y se realizó la eliminación de la vaina con hipoclorito de sodio al $0,187 \%$ por cinco minutos y posteriormente se realizaron tres lavados con agua destilada por centrifugación a $2.000 \mathrm{rpm}$ a $4{ }^{\circ} \mathrm{C}$ por $5 \mathrm{~min}$. El pellet de las $\mathrm{L}_{3}$ fue suspendido en agua destilada para su cuantificación por alícuotas.

Para los bioensayos se utilizó la mezcla comercial de albendazol + closantel (alb+clos) $\left(37,5 \mathrm{mg} / \mathrm{mL}^{-1}\right.$ y
$50 \mathrm{mg} / \mathrm{mL}^{-1}$, respectivamente), ivermectina $\left(10 \mathrm{mg} / \mathrm{mL}^{-1}\right)$ y levamisol al $12 \%$. La evaluación de la acción antihelmíntica se realizó por duplicado en cajas de poliestireno de 96 pozos, con $100 \mathrm{~L}_{3}$ por pozo y $100 \mu \mathrm{l}$ de cada antihelmíntico en diluciones con agua destilada (cuadro 1).

La lectura de $\mathrm{L}_{3}$ vivas o muertas se realizó a las 12 y $24 \mathrm{~h}$ postinteracción con el tratamiento (antihelmíntico) y también con un testigo positivo (agua destilada). Los datos obtenidos fueron analizados para obtener la concentración letal $\left(\mathrm{CL}_{50}\right)$ y la dosis letal $\left(\mathrm{DL}_{50}\right)$ por fármaco y por aislamiento mediante el análisis Probit, utilizando el programa SAS (SAS 1999) sobre la base del siguiente modelo:

$$
\begin{gathered}
\operatorname{Pr}(\text { Respuesta })=\mathrm{C}+(1-\mathrm{C}) \mathrm{F}\left(\mathrm{X}^{\prime} \beta\right)=\mathrm{C}+(1-\mathrm{C}) \Phi\left(\mathrm{b}_{0}+\mathrm{b}_{1}\right. \\
\left.\mathrm{x} \log _{10}(\text { Dosis })\right)
\end{gathered}
$$

Donde:

$\beta$ es un vector de parámetros estimados

F es una función de distribución acumulativa (normal)

$\mathrm{X}$ es un vector de variables explicativas

Pr es la probabilidad de una respuesta

$\mathrm{C}$ es la tasa de respuesta natural (proporción de individuos que responden a la dosis cero).

\section{RESULTADOS}

\section{EFICACIA Y RESISTENCIA A LOS ANTIHELMÍNTICOS}

Los datos obtenidos indican que el levamisol mostró bajo porcentaje de eficacia antihelmíntica $(30,2 \%$ de reducción en el conteo de hpg) postratamiento. En contraste, con albendazol y con la aplicación comercial de

Cuadro 1. Esquema de la concentración de tres antihelmínticos utilizados en contra de $\mathrm{L}_{3}$ de Haemonchus contortus y Cooperia curticei del aislamiento Ch y Haemonchus contortus aislamiento CENID.

Concentration of three anthelmintics used against Haemonchus contortus and Cooperia curticei Chapingo isolation and Haemonchus contortus CENID isolation.

\begin{tabular}{cccc}
\hline Diluciones & $\begin{array}{c}\text { Albendazol + closantel } \\
\left(\mathrm{mg} / \mathrm{mL}^{-1}\right)\end{array}$ & $\begin{array}{c}\text { Ivermectina } \\
\left(\mathrm{mg} / \mathrm{mL}^{-1}\right)\end{array}$ & $\begin{array}{c}\text { Levamisol } \\
\left(\mathrm{mg} / \mathrm{mL}^{-1}\right)\end{array}$ \\
\hline Comercial & $37,5+50$ & 10 & 120 \\
2 & $18,75+25$ & 5 & 60 \\
3 & $9,38+12,5$ & 3 & 30 \\
4 & $4,69+6,25$ & 1,25 & 15 \\
5 & $2,34+3,13$ & 0,63 & 7,5 \\
6 & $1,17+1,56$ & 0,31 & 3,75 \\
7 & $0,59+0,78$ & 0,16 & 1,88 \\
8 & $0,29+0,39$ & 0,08 & 0,94 \\
9 & $0,15+0,20$ & 0,04 & 0,47 \\
10 & $0,073+0,10$ & 0,02 & 0,23 \\
11 & $0,037+0,05$ & 0,01 & 0,12 \\
12 & $0,018+0,024$ & 0,005 & 0,06 \\
\hline
\end{tabular}


Cuadro 2. Eficacia de tres antihelmínticos en el control de nematodos gastrointestinales de ovinos de pelo. Effectiveness of three anthelmintics for gastrointestinal nematode control of hair sheep.

\begin{tabular}{|c|c|c|c|c|c|}
\hline Antihelmíntico & Fecha de aplicación & Número de ovejas & HPG previo & HPG posterior & Eficacia $(\%) *$ \\
\hline Ivermectina (Iv) & Junio-Julio & 14 & $3.725^{\mathrm{a}}$ & $429^{b}$ & $86,7^{\mathrm{a}} \pm 20,1$ \\
\hline Albendazol & Agosto-Septiembre & 26 & $2.038^{\mathrm{a}}$ & $565^{\mathrm{b}}$ & $64,0^{\mathrm{ab}} \pm 44,6$ \\
\hline Levamisol (L) & Octubre & 7 & $2.464^{\mathrm{a}}$ & $1.729^{\mathrm{a}}$ & $30,2^{\mathrm{c}} \pm 39,0$ \\
\hline $\mathrm{Iv}+\mathrm{L}$ & Diciembre & 7 & $3.021^{\mathrm{a}}$ & $1.293^{\mathrm{ab}}$ & $65,0^{\mathrm{bc}} \pm 36,6$ \\
\hline
\end{tabular}

* La separación de medias corresponde a la raíz cuadrada de la variable (hpg después de la desparasitación/hpg antes de la desparasitación).

ivermectina + levamisol se registró un valor mayor de eficacia (65\%; cuadro 2). La ivermectina mostró $87 \%$ de eficacia al administrarse como producto único y en todos los casos se observó alta variabilidad en la reducción de los conteos de hpg (desviación estándar, DE, superior a $20 \%$ en la eficacia).

En la prueba de resistencia con la aplicación de Iv+L, se observó $51 \%$ eficacia (antes y después de la aplicación) y considerando los lineamientos de la WAAVP solo hubo una diferencia de $1,3 \%$ de reducción en el hpg en el grupo tratado respecto del grupo control, por lo que de acuerdo a los criterios de la WAAVP se determinó la existencia de resistencia antihelmíntica a la aplicación conjunta de Iv+L (cuadro 3).

\section{PRUEBAS IN VITRO}

En el estudio in vitro el análisis Probit mostró que en casi todos los ensayos la ordenada al origen $\left(b_{0}\right)$ y la pendiente de la regresión $\left(\mathrm{b}_{1}\right)$ fueron significativas $(\mathrm{P}<0,05)$, excepto en el aislamiento $\mathrm{Ch}$ tratado con ivermectina y leído a las 12 horas. En las ecuaciones Probit se observaron tasas de mortalidad natural entre 12 a $23 \%$ (C) y para el resto de las larvas (1-C) la pendiente de la regresión $\left(\mathrm{b}_{1}\right)$ fue
Cuadro 3. Resultados de la prueba de resistencia antihelmíntica en ovinos desparasitados conjuntamente con levamisol e ivermectina.

Results of anthelmintic resistance test in hair sheep, drenched together with levamisole and ivermectin.

\begin{tabular}{lcc}
\hline Variable & Control & Tratado \\
\hline Número de ovinos & 8 & 8 \\
Conteo de hpg pretratamiento & 956 & 1937 \\
Conteo de hpg postratamiento & 963 & 950 \\
Porcentaje de reducción (eficacia) & 51 \\
Porcentaje de reducción respecto del control & 1,3 \\
Varianza de la red & 0,05 \\
Límite de confianza superior & 36,7 \\
Límite de confianza inferior & $-53,9$ \\
\hline
\end{tabular}

positiva en todos los casos, por lo que con el incremento en la concentración del antihelmíntico aumentó la tasa de mortalidad (cuadro 4).

Con albendazol + closantel se observó que la $\mathrm{DL}_{50}$ fue mayor en el aislamiento Ch (albendazol + closantel: 38,8 y $51,7 \mathrm{mg} / \mathrm{mL}^{-1}$ ) cuando se observaron las larvas a las doce horas, en comparación con los ngi provenientes

Cuadro 4. Ecuaciones Probit, coeficientes de regresión y concentración letal de tres antihelmínticos utilizados en el control de nematodos gastrointestinales.

Probit equations, regression coefficients and lethal doses of three anthelmintics used for gastrointestinal nematodes control.

\begin{tabular}{|c|c|c|c|c|c|c|}
\hline Antihelmíntico & Tiempo & Ecuación Probit & $\mathrm{b}_{0}$ & $\mathrm{~b}_{1}$ & $\mathrm{CL}_{50}$ & $\mathrm{DL}_{50}$ \\
\hline \multicolumn{7}{|l|}{ Aislamiento CENID } \\
\hline $\begin{array}{l}\text { Albendazol }(\mathrm{Ab})+ \\
\text { Closantel }(\mathrm{C})^{*}\end{array}$ & 12 & $\operatorname{Pr}=0,22+0,78(\varphi(-3,98+3,06 \times \log 10($ dosis $)))$ & * & $* *$ & 1,07 & 11,86 \\
\hline Ivermectina & 12 & $\operatorname{Pr}=0,23+0,77(\varphi(1,29+1,14 \times \log 10($ dosis $)))$ & $* *$ & $* *$ & $-1,38$ & 0,042 \\
\hline Levamisol & 12 & $\operatorname{Pr}=0,19+0,81(\varphi(-4,47+3,57 \times \log 10($ dosis $)))$ & $* *$ & $* *$ & 1,11 & 12,87 \\
\hline \multicolumn{7}{|l|}{ Aislamiento $\mathrm{Ch}$} \\
\hline $\mathrm{Ab}+\mathrm{C}^{*}$ & 12 & $\operatorname{Pr}=0,13+0,87(\varphi(-4,14+3,13 \times \log 10($ dosis $)))$ & $* *$ & $* *$ & 1,59 & 38,79 \\
\hline $\mathrm{Ab}+\mathrm{C}^{*}$ & 24 & $\operatorname{Pr}=0,17+0,83(\varphi(-8,53+8,02 \times \log 10($ dosis $)))$ & $* *$ & $* *$ & 0,95 & 8,88 \\
\hline Ivermectina & 12 & $\operatorname{Pr}=0,15+0,85(\varphi(4,37+21,80 \times \log 10($ dosis $)))$ & ns & ns & $-0,39$ & 0,41 \\
\hline Ivermectina & 24 & $\operatorname{Pr}=0,23+0,77(\varphi(1,62+0,60 \times \log 10($ dosis $)))$ & $* *$ & $* *$ & $-3,25$ & 0,0006 \\
\hline Levamisol & 12 & $\operatorname{Pr}=0,12+0,88(\varphi(-3,29+2,86 \times \log 10($ dosis $)))$ & $* *$ & $* *$ & 0,98 & 9,57 \\
\hline Levamisol & 24 & $\operatorname{Pr}=0,13+0,87(\varphi(-2,47+2,28 \times \log 10($ dosis $)))$ & $* *$ & $* *$ & 0,92 & 8,23 \\
\hline
\end{tabular}

* La concentración indicada es solo para albendazol, la correspondiente para closantel estará de acuerdo con el cuadro 1. 
del CENID (albendazol + closantel: 11,9 y 15,9 mg/mL ${ }^{-1}$ ). Sin embargo, en la lectura a las 24 horas en el aislamiento $\mathrm{Ch}$, la $\mathrm{DL}_{50}$ se redujo hasta un valor de 8,9 y $11,9 \mathrm{mg} \mathrm{ml}^{-1}$ para albendazol + closantel, con lo que se requirió de una dosis menor para reducir en 50\% la población de nematodos cuando el tiempo de exposición fue mayor (figura 1).

Cuando se comparó la aplicación de ivermectina en los dos aislamientos a un mismo tiempo (12 horas), la $\mathrm{DL}_{50}$ fue mayor en el aislamiento $\mathrm{Ch}\left(0,41 \mathrm{mg} \mathrm{ml}^{-1}\right)$ que en las larvas del CENID $\left(0,04 \mathrm{mg} \mathrm{ml}^{-1}\right)$. Sin embargo, cuando se dejó actuar el producto por 12 horas más en el aislamiento $\mathrm{Ch}$, la $\mathrm{DL}_{50}$ fue menor (figura 2)

Para el aislamiento CENID únicamente con levamisol en la lectura a las 12 horas se observó una mayor $\mathrm{DL}_{50}$ respecto de la determinada para el aislamiento $\mathrm{Ch}$ a las 12 y a las 24 horas (figura 3 ).

\section{DISCUSIÓN}

El bajo efecto nematicida de levamisol en el rebaño de Salto de Agua, Chiapas (30\% de eficacia), se atribuyó al uso constante de este antihelmíntico durante un periodo cercano a cuatro años. La baja eficacia ya se había indicado previamente en un estudio, en el que se utilizaron tres antihelmínticos (levamisol, ivermectina y albendazol + closantel), con la intención de reducir el número de huevos de ngi en ovinos en pastoreo (González-Garduño y col 2010).

Con ivermectina el promedio de eficacia fue de $87 \%$ cuando se utilizó al inicio del periodo de evaluación. Sin embargo, debido al comportamiento variable entre las ovejas $(\mathrm{DE}=20 \%)$ se suspendió su uso y cuando se volvió a utilizar de manera conjunta con levamisol, la reducción en el conteo fecal de huevos de ngi fue menor (65\%) y con mayor variabilidad en la reducción del conteo de huevos de ngi ( $\mathrm{DE}=36,6 \%)$. La varianza en la eficacia implica que en algunas ovejas los conteos fecales de huevos de ngi se redujeron, pero en otras en vez de disminuir se incrementaron, y en un caso una oveja a pesar de haberse desparasitado con levamisol presentó anemia, anorexia y finalmente murió, ello se atribuyó a la presencia de $H$. contortus y $C$. curticei observados a la necropsia. Esta variabilidad en la eficacia también se ha indicado en compuestos derivados de bencimidazol en un estudio reciente (Muñoz y col 2008). Sin embargo, esos autores indicaron alta eficacia para ivermectina y doramectina, con una persistencia del efecto antihelmíntico durante 35 días. Aunque también existen indicios actuales de resistencia contra ivermectina en el estado de Tlaxcala (MontalvoAguilar y col 2006). A pesar de que no fue posible separar la resistencia por especie, se pudo determinar en la necropsia que esta ocurre tanto en $H$. contortus como en $C$. curticei, los que son los dos principales nematodos encontrados en la unidad de producción. Esto se confirmó en los cultivos larvarios del rebaño. Sin embargo, es necesario hacer estudios independientes para cada especie para comprobar el grado de resistencia de cada una de ellas.

Debido a la baja eficacia existente en todos los productos utilizados en las ovejas, se decidió realizar una prueba de resistencia a la combinación de ivermectina y levamisol. Sin embargo, a pesar de utilizarse de manera conjunta no fue posible reducir los conteos de hpg en todos los animales y se obtuvieron resultados similares

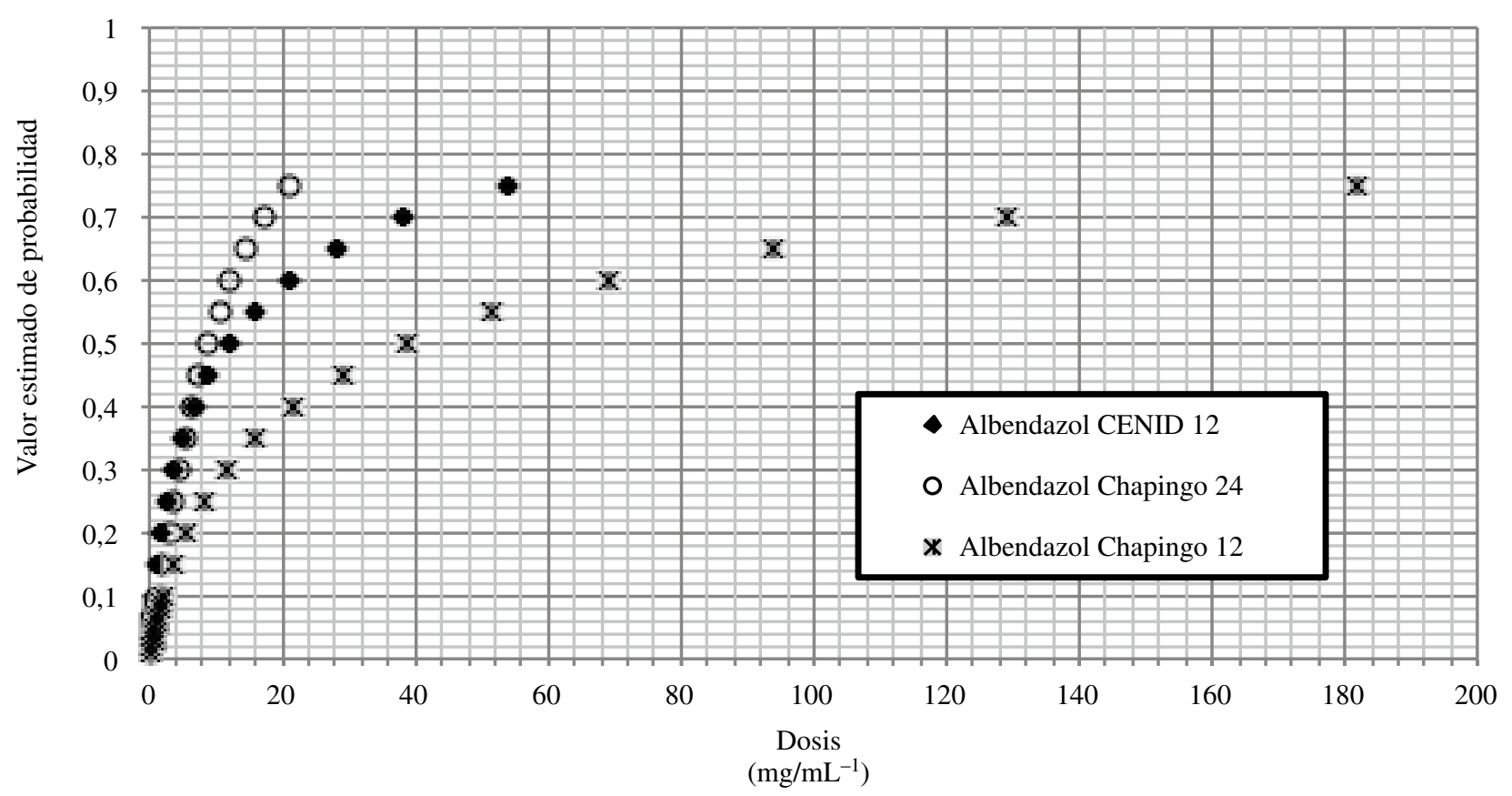

Figura 1. Resultado comparativo de la mortalidad de larvas infectantes de nematodos gastrointestinales de ovinos utilizando albendazol + closantel.

Comparative results in mortality of gastrointestinal nematodes infective larvae of sheep, using albendazole + closantel. 


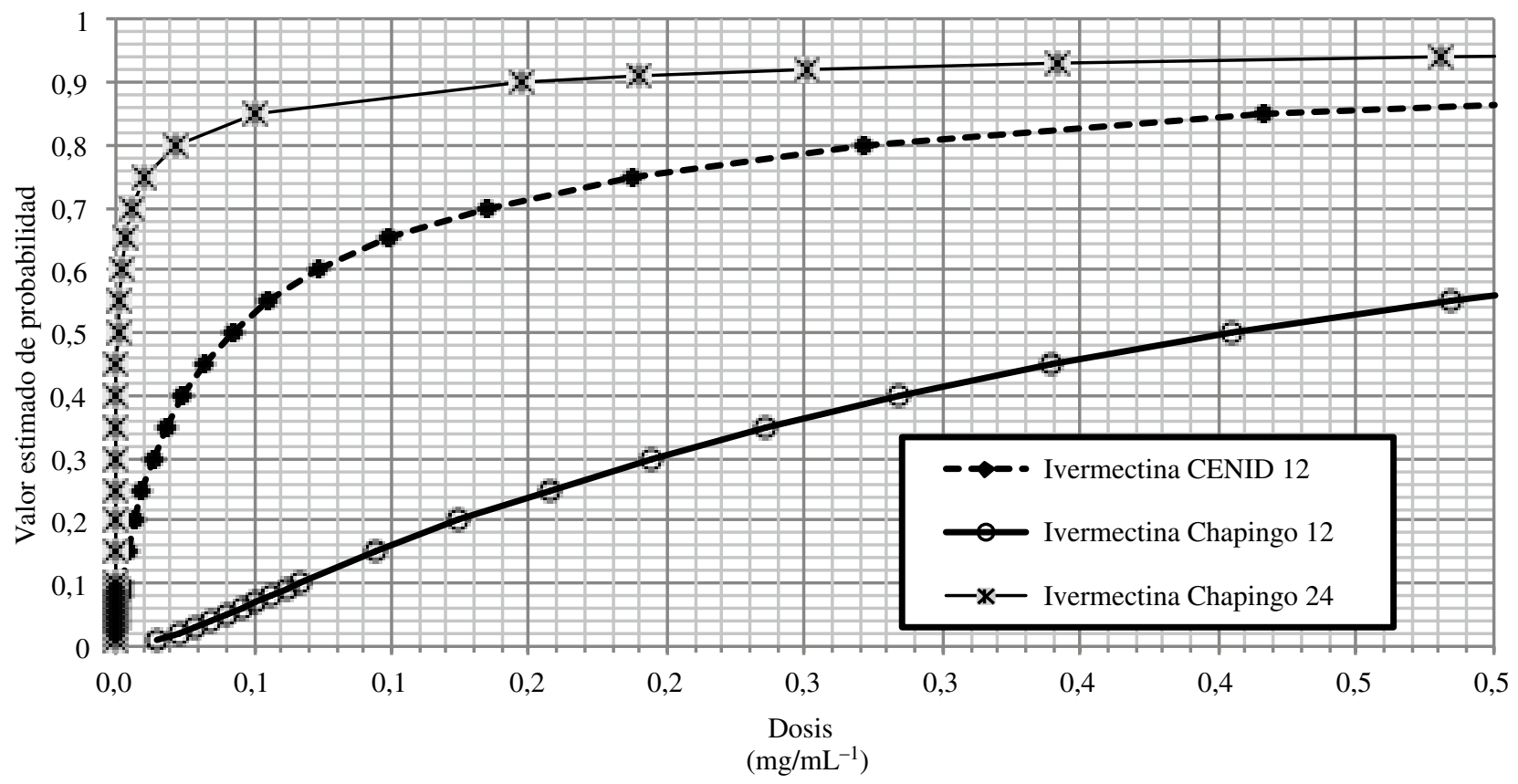

Figura 2. Resultado comparativo de la mortalidad de larvas de nematodos gastrointestinales de ovinos de pelo utilizando ivermectina. Comparative results in mortality of gastrointestinal nematodes infective larvae of hair sheep, using ivermectin.

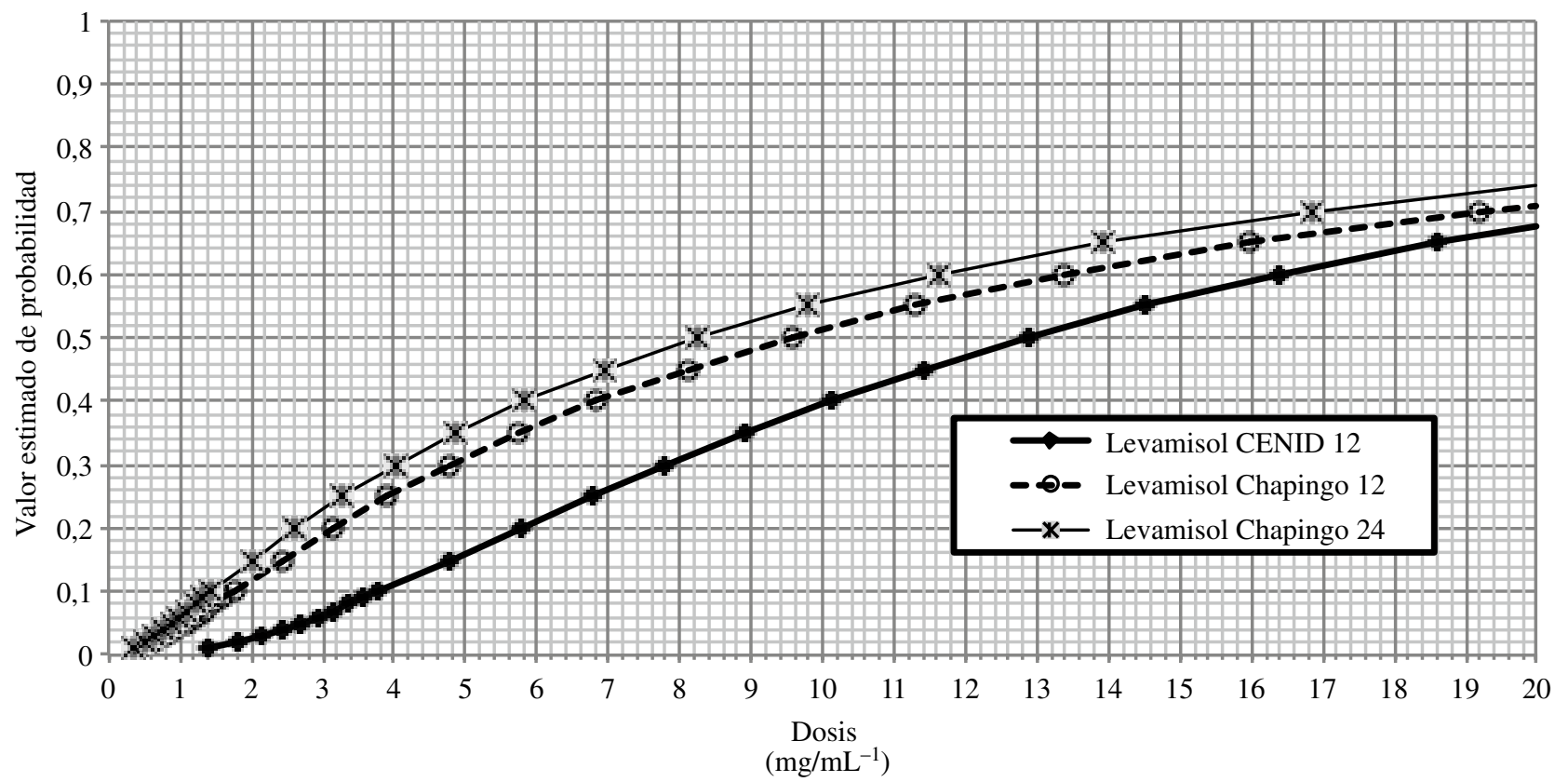

Figura 3. Resultado comparativo de la mortalidad de larvas de nematodos gastrointestinales de ovinos de pelo utilizando levamisol. Comparative results in mortality of gastrointestinal nematodes infective larvae of hair sheep, using levamisole.

a los de la mezcla de albendazol + levamisol aplicada en otro estudio (Márquez 2003).

El rápido incremento en la resistencia desarrollada por lo ngi contra ivermectina se ha demostrado en $H$. contortus, en un estudio en el que al cabo de tres generaciones de larvas la eliminación fecal de huevos de esta especie se reducía a 65\% al usar el producto (Coles y col 2005). También en Nicaragua se han obtenido algunos indicios de resistencia antihelmíntica con la prueba de PRCFH con ivermectina y levamisol (Rimbaud y col 2005) y en Chile se ha indicado resistencia antihelmíntica en bovinos (Sievers y Alocilla 2007).

En el análisis Probit no se encontró significancia en la ordenada al origen $\left(b_{0}\right)$ y en la pendiente de la regresión $\left(b_{1}\right)$ en el aislamiento Ch tratado con ivermectina, lo que indica que no hubo relación directa entre la dosis y la mortalidad 
larvaria, esto es posiblemente consecuencia de la baja eficacia y la gran variación existente en la reducción del conteo fecal de huevos de ngi entre los animales, aunque con mayor tiempo de exposición se pudo observar que sí hubo relación entre el tiempo y la mortalidad ocurrida (pendiente de la curva).

En todos los casos la dosis letal requerida fue superior en los nematodos gastrointestinales del aislamiento Ch que en las larvas de $H$. contortus resistentes a bencimidazol proveniente del CENID, por lo que existen indicios para suponer que el aislamiento Ch tiene ngi con un alto grado de resistencia múltiple. Los resultados del PRCFH obtenidos en este estudio fueron confirmados con los estudios de las pruebas in vitro, tomando como referencia el aislamiento resistente del CENID.

La resistencia en los aislamientos observados se podría abatir si se aplican dosis mayores de $9,6 \mathrm{mg} / \mathrm{ml}^{-1}$ para levamisol, $0,41 \mathrm{mg} / \mathrm{ml}^{-1}$ para ivermectina y $39 \mathrm{mg} /$ $\mathrm{ml}^{-1}$ para albendazol. El incremento de dosis es un proceso que se ha seguido para el control de nematodos en poblaciones con alto índice de resistencia, sin embargo, también representa un problema al generar otro tipo de mutaciones múltiples que puedan estar fuera de control en los programas sanitarios (Márquez 2003). Por ello se recomienda utilizar otras herramientas de control de nematodos gastrointestinales y de manera integral con uso cuidadoso de los antihelmínticos comerciales.

El uso constante de levamisol ha originado una reducida eficacia (30\%) en el control de nematodos gastrointestinales ( $H$. contortus y $C$. curticei) en ovinos de pelo. Por otra parte la combinación de levamisol con invermectina mostró resistencia antihelmíntica en la prueba PRCFH y también la combinación albendazol + closantel mostró resistencia, comprobada con la prueba de motilidad larvaria in vitro, la que representa una herramienta de diagnóstico más sensible para evaluar diferentes antihelmínticos en poblaciones de nematodos.

\section{AGRADECIMIENTOS}

Al Sr. Natael Guillén García por su valioso apoyo en los muestreos y en el manejo del rebaño. A José Arias Julián y Demetrio Esteban Andrés por su ayuda en el procesamiento de las muestras.

\section{REFERENCIAS}

Arece J, M Mahieu, H Archimède, G Aumont, M Fernández, E González, O Cáceres, A Menéndez-Buxadera. 2004. Comparative efficacy of six anthelmintics for the control of gastrointestinal nematodes in sheep in Matanzas, Cuba. Small Rumin Res 54, 61-67.

Chartier C, I Pors, J Hubert, D Rocheteau, C Benoit, N Bernard. 1998. Prevalence of anthelmintic resistant nematodes in sheep and goats in western France. Small Rumin Res 29, 33-41.

Coles GC, C Bauer, FHM Borgsteede, S Geerts, TR Klei, MA Taylor, PJ Waller. 1992. World Association for the Advancement of Veterinary Parasitology (WAAVP) methods for the detection of anthelmintic resistance in nematodes of veterinary importance. Vet Parasitol 44, 35-44.

Coles GC, AC Rhodes, AJ Wolstenholme. 2005. Rapid selection for ivermectin resistance in Haemonchus contortus. Vet Parasitol 129, 345-347.
Coles GC, F Jackson, WE Pomroy, RK Prichard, G von SamsonHimmelstjerna, A Silvestre, MA Taylor, J Vercruysse. 2006. The detection of anthelmintic resistance in nematodes of veterinary importance. Vet Parasitol 136, 167-185.

Encalada-Mena LA, ME López-Arellano, P Mendoza-de Gives, E Liébano-Hernández, V Vázquez-Prats, G Vera-Ycuspinera. 2008. Primer informe en México sobre la presencia de resistencia a ivermectina en bovinos infectados naturalmente con nematodos gastrointestinales. Vet Méx 39, 423-428.

García E. 1988. Modificaciones al Sistema de Clasificación Climática de Köppen. $4^{\mathrm{a}}$ ed. Editorial Instituto de Geografía, Universidad Nacional Autónoma de México, México, Pp 90-95.

González-Garduño R, JC Cordero-Ortega, G Torres-Hernández, J AreceGarcía, P Mendoza-de Gives. 2010. Efecto del hipoclorito de sodio y extracto de cítricos en la reducción de la infestación con nematodos gastrointestinales resistentes a antihelmínticos en ovinos de pelo. Rev Mex Cienc Pecu 1, 179-187.

Jabbar A, Z Iqbal, D Kerboeuf, G Muhammad, MN Khan, M Afaq. 2006. Anthelmintic resistance: The state of play revisited. Life Sci 79, 2413-2431.

Kochapakdee S, VS Pandey, W Pralomkarn, S Choldumrongkul, W Ngampongsai, A Lawpetchara. 1995. Anthelmintic resistance in goats in southern Thailand. Vet Rec 137, 124-125.

Márquez LD. 2003. Resistencia a los antihelmínticos: origen, desarrollo y control. Rev Corpoica 4, 55-71.

Molento MB. 2009. Parasite control in the age of drug resistance and changing agricultural practices. Vet Parasitol 163, 229-234.

Montalvo-Aguilar X, ME López-Arellano, V Vázquez-Pratts, E LiébanoHernández, P Mendoza-de Gives. 2006. Resistencia antihelmíntica de nematodos gastroentéricos en ovinos a febendazol e ivermectina en la región noroeste del estado de Tlaxcala. Téc Pec Méx 44, 81-90.

Muñoz JA, C Angulo, R Ramírez, O Vale, E Chacín, D Simoes, A Atencio. 2008. Eficacia antihelmíntica de doramectina $1 \%$, ivermectina $1 \%$ y ricobendazol 15\% frente a nematodos gastrointestinales en ovinos de pelo. Rev Científica FCV-LUZ 18, 12-16.

Papadopoulos E. 2008. Anthelmintic resistance in sheep nematodes. Small Rumin Res 76, 99-103.

Rimbaud E, P Zúniga, M Doña, N Pineda, L Luna, G Rivera, L Molina, J Gutiérrez, J Vanegas. 2005. Primer diagnóstico de resistencia a levamisol y lactonas macrocíclicas en nematodos gastrointestinales parásitos de ovinos en Nicaragua. Red Vet VI, 1-3.

Sangster NC. 2001. Managing parasiticide resistance. Vet Parasitol 98, 89-109.

SAS Institute. 1999. The SAS System for Windows. Version 8. SAS Institute. Inc. Cary, N. C. USA.

Sievers G, A Alocilla. 2007. Determinación de resistencia antihelmíntica frente a ivermectina de nematodos del bovino en dos predios del sur de Chile. Arch Med Vet 39, 67-69.

Taylor MA, KR Hunt, KL Goodyear. 2002. Anthelmintic resistance detection methods. Vet Parasitol 103, 183-194.

Thienpont D, F Rochette, OFJ Vanparijs. 1986. Diagnóstico de las helmintiasis por medio del examen coprológico. Janssen Research Foundation, Beerse, Bélgica, Pp 19-43.

Torres-Acosta JFJ, U Dzul-Canche, AJ Aguilar-Caballero, RI RodríguezVivas. 2003. Prevalence of benzimidazole resistant nematodes in sheep flocks in Yucatan, Mexico. Vet Parasitol 114, 33-42.

Vilaboa-Arroniz J, R Bozzi, P Díaz-Rivera, L Bazzi. 2010. Conformación corporal de las razas ovinas Pelibuey, Dorper y Kathadin en el estado de Veracruz, México. Zoot Trop 28, 321-328.

Von Samson-Himmelstjerna G, W Blackhall. 2005. Will technology provide solutions for drug resistance in veterinary helminths? Vet Parasitol 132, 223-239.

Wagland BM, WO Jones, L Hribar, T Bendixsen, DL Emery. 1992. A new simplified assay for larval migration inhibition. Int J Parasitol $22,1183-1185$. 